\begin{tabular}{|c|c|}
\hline \multicolumn{2}{|c|}{ Statistica Sinica Preprint No: SS-2021-0246 } \\
\hline Title & $\begin{array}{l}\text { Partially Functional Linear Quantile Regression With } \\
\text { Measurement Errors }\end{array}$ \\
\hline Manuscript ID & SS-2021-0246 \\
\hline URL & http://www.stat.sinica.edu.tw/statistica/ \\
\hline DOI & $10.5705 /$ ss.202021.0246 \\
\hline Complete List of Authors & $\begin{array}{l}\text { Mengli Zhang, } \\
\text { Lan Xue, } \\
\text { Carmen D. Tekwe, } \\
\text { Yang Bai and } \\
\text { Annie Qu } \\
\end{array}$ \\
\hline Corresponding Author & Lan Xue \\
\hline E-mail & xuel@science.oregonstate.edu \\
\hline tice: Accepted version subjec & ct to English editing. \\
\hline
\end{tabular}


Statistica Sinica

\title{
PARTIALLY FUNCTIONAL LINEAR QUANTILE REGRESSION WITH MEASUREMENT ERRORS
}

\author{
Mengli Zhang, Lan Xue, Carmen D. Tekwe, Yang Bai and Annie Qu \\ Shanghai University of Finance and Economics, Oregon State University, \\ Indiana University School of Public Health and University of California, Irvine
}

\begin{abstract}
Ignoring of measurement errors in conventional regression analyses can lead to biased estimation and inference results. Reducing such bias could be challenging when the error-prone covariate is a functional curve. In this paper, we propose a new corrected loss function for partially functional linear quantile model with function-valued measurement errors. We establish asymptotic properties of both functional coefficient and parametric coefficient estimators. We also demonstrate finite sample performance of the proposed method through simulation studies, and illustrate its advantages for children obesity study.
\end{abstract}

Key words and phrases: Corrected score; Functional principle component; Functional measurement error; Physical activity; Quantile regression; Wearable devices.

\section{Introduction}

Wearable devices are increasingly used in health research to monitor health and well-being, and can be combined with lifestyle interventions to reduce obesity. Wearable devices provide continuous granulated measurements of physical 
activity (PA) with raw PA measured at a sub-second level. These measurements can be viewed as functions or curves, rather than as vectors. Although recent developments in functional data analysis (FDA) can be applied to such data, there are remaining analytical challenges need to be solved so that more accurate inferences can be drawn in obesity prevention studies. First, standard analytical methods employed in current obesity studies apply regression approaches to model the mean BMI, which could potentially limit the detection of intervention effects among participants whose weights differ from the mean (Koenker, 2005, Geraci and Bottai, 2014). Second, because true patterns of PA behavior are not directly observable, wearable devices are used to monitor PA, thus introducing measurement error. Sources of measurement error associated with wearable-device-based measures of PA include variability in prediction at various PA intensity levels and errors associated with the prediction equations (Bassett, 2012; Crouter et al., 2006; Jacobi et al., 2007; Warolin et al., 2012; Rothney et al., 2008). Failure to account for measurement error in assessing the effects of PA measures on health outcomes often leads to bias and the underestimation of these effects (Tekwe et al., 2019). Third, data collected from wearable devices are typically not discrete vectors but rather a curvilinear functions of time, and require the use of functional data approaches in (Silverman and Ramsay, 2005).

While researchers have studied measurement error in traditional multivari- 
ate data analysis extensively (Carroll et al., 2006; Fuller, 1987), there has been limited work on measurement error in the context of FDA. When conducting FDA, it is usually assumed that measurement errors are independent and identically distributed. However, this assumption is not feasible because the functional measurement errors tend to be correlated over time in practice. Therefore, there is a need to take functional measurement errors with a more complex error structures into account in functional data analysis. In this paper, we will address these critical needs and analytical challenges, and the proposed method provides a better understanding of how measurement error could impact the evaluation of wearable-device-based PA patterns in obesity studies.

Our proposed research is motivated by a childhood obesity study in which stand-biased desks were introduced to schools as an intervention to increase school day physical activity. The original research goal was to assess the association between daily energy expenditure (DEE) and subsequent progression toward obesity. Since DEE is not directly observable, the investigators provide the children accelerometer armbands to estimate their DEE approximately. In this project, we will develop statistical methods to model measurements of DEE obtained from wearable devices as functional data prone to measurement error, and also reduce the impacts of measurement error in DEE for predicting obesity. In addition, our methods are based on quantile regression and focus on the entire 
distribution of body mass index (BMI); rather than just average BMI values.

Quantile regression (Koenker and Bassett, 1978) has emerged as an important statistical method that offers a systematic approach for examining the effects of covariates on the entire distribution of the response variable, in contrast with traditional mean regression. Researchers have recently applied quantile regression to functional data. Kato (2012) studied estimation in functional linear quantile model and derived the rate of convergence for the functional coefficient estimator. Yao et al. (2017) proposed a regularized partially linear functional quantile regression for simultaneous estimation and selection of the important covariates. Both aforementioned papers assume the functional covariate is exactly observed at subject-specific sampling points.

When covariates are not directly observable and instead measured with errorprone proxies, calibrating measurement error in the quantile regression framework is challenging for two reasons. First, a parametric assumption of the regression error distribution is often unavailable in quantile regressions. Second, the quantile function, unlike the mean function, does not inherit the additive property (Wang et al., 2012). As a result, limited studies regarding measurement error in quantile regression settings have been investigated.

Recent progresses on this topic include Wang et al. (2012); Wei and Carroll (2009); He and Liang (2000); Hu and Schennach (2008). However, all 
aforementioned methods are restricted to cases when the covariates are scalars of finite dimensions. In particular, He and Liang (2000) introduced a consistent estimation procedure based on orthogonal residuals under the assumption that model error and measurement error follow a common distribution which is spherically symmetric. Wei and Carroll (2009) introduced joint estimating equations that simultaneously hold for all the quantile levels, where a general linear regression structure assumption for all conditional quantiles is needed. Besides, their method requires to estimate conditional density of the responses given independent variables. Firpo et al.(2017) proposed a semi-parametric twostep estimator to improve the strong linearity assumption on all quantile levels and the iterative algorithm in Wei and Carroll (2009). However, this requires non-parametric estimations of the conditional density of true variables given response and other error-free covariates. It would be difficult to obtain such estimates when the dimensionality of error-prone covariates is large. By contrast, Wang et al. (2012) constructed a corrected-loss estimation only at specific quantile levels and avoided the strong assumption of mutual symmetric error or non-parametric estimation of the conditional densities.

The idea of correction for loss/score functions in the context of covariate measurement errors was first introduced by Nakamura (1990), where the corrected log-likelihood/score function was constructed as an unbiased estimator of 
the conditional expectation of original log likelihood/score function given measurement errors. Estimating equations were also derived for generalized linear models with normal measurement errors. However, the non-differentiable loss function in quantile regression makes it difficult to construct such equations. Wang et al. (2012) addressed this issue by using a smooth function to approximate the indicator function, and the corrected loss function was derived under multivariate normal or Laplace distributions of measurement errors.

In this paper, we solve a more challenging problem when the covariate of interest is functional and the associated unknown regression coefficient is nonparametric and of infinite dimension. This scenario brings significant difficulties for developing algorithms and deriving the asymptotic properties of the proposed methods. To our knowledge, our paper is the first attempt to address the functionvalued measurement error in functional quantile regression models. In this paper, we propose a corrected loss approach in Wang et al. (2012) for partially functional linear quantile regression when the functional covariate is contaminated with functional measurement errors. In particular, our method identifies the measurement error model by assuming a parametric form for the measurement error distribution and constructs a corrected objective function using a class of smoothed quantile objective functions. The proposed method provides consistent estimator of functional regression coefficient and asymptotically normal 
estimates of parametric coefficients. In addition, our method does not require specification of the distribution for regression error.

The rest of paper is organized as follows. Section 2 introduces the partially functional quantile model. Corrected loss and practical implementation are demonstrated in Section 3. Section 4 establishes asymptotic property of our proposed estimators. Simulation studies in Section 5 are performed to show finite sample performance of the proposed method. Section 6 contains a real data application from a children obesity study. Our conclusion is provided in Section 7. The technical lemmas and proofs are included in the supplementary material.

\section{Background and notations}

Suppose $\left(Y_{i}, X_{i}, \mathbf{Z}_{i}\right)_{i=1}^{n}$ are independent realizations from the distribution of $(Y, X, \mathbf{Z})$, where $Y$ is a scalar random variable, and $X=\{X(t), t \in \mathcal{T}\}$ is a random function assumed to be square integrable on a bounded closed interval $\mathcal{T}$ in $\mathbb{R}$ and possibly contaminated with measurement errors. Without loss of generality, we assume $\mathcal{T}=[0,1]$, and $X$ is centered with $E[X(t)]=0$ for $t \in \mathcal{T}$. Here $\mathbf{Z}$ is a $p$ dimensional vector of error-free covariates including the intercept term. For a given $\tau \in(0,1)$, the $\tau$-th conditional quantile function $Q_{Y \mid X, \mathbf{Z}}(\tau)$ is defined as $F_{Y \mid X, \mathbf{Z}}^{-1}(\tau)$, where $F_{Y \mid X, \mathbf{Z}}(y)=P(Y \leq y \mid X, \mathbf{Z})$ is the cumulative distribution function of $Y$ conditional on $X$ and $\mathbf{Z}$. For $i=1, \ldots, n$, 
we assume

$$
Q_{Y_{i} \mid X_{i}, \mathbf{Z}_{i}}(\tau)=\mathbf{Z}_{i}^{T} \boldsymbol{\theta}_{0}(\tau)+\int_{0}^{1} \beta_{0}(t, \tau) X_{i}(t) d t
$$

where $\boldsymbol{\theta}_{0}(\tau)$, including a scalar-valued intercept term, represents the vector of coefficients associated with the error-free covariates for the $\tau^{\text {th }}$ quantile, while $\beta_{0}(t, \tau) \in L_{2}[0,1]$ is a functional coefficient that quantifies the effect of the functional covariate. For each individual $i$, we assume the functional covariate $X_{i}$ is not directly observable, instead it is approximated by a surrogate $W_{i}$ as

$$
W_{i}(t)=X_{i}(t)+U_{i}(t)
$$

Here $W_{i}$ serves as unbiased measures of $X_{i}$ subject to a functional measurement error $U_{i}(t)$, where $E\left[U_{i}(t)\right]=0$ for $t \in \mathcal{T}$ and $U_{i}$ is independent of $X_{i}, \mathbf{Z}_{i}$ and $Y_{i}$. The existing literature on functional data often assumes the measurement errors $U_{i}(t)$ are independent and identically distributed (i.i.d) with a common variance over time $t$. Here we consider a functional measurement error and allow $U_{i}(t)$ to have an unstructured covariance function $\Sigma_{U}(t, s)$. When $U_{i}(t)$ are i.i.d., its common variance can be estimated using $\left\{W_{i}(t)\right\}$ under the smoothness of the covariance function of $X_{i}(t)$, which is similar to the estimation of nugget effect in spatial statistics. However, when $\Sigma_{U}(t, s)$ is unstructured, additional 
information is needed to identify the covariance structure of $U_{i}(t)$, and measurement errors need to be taken into account to ensure consistent estimation and inference of the partially functional linear quantile regression model (1.1).

The partially functional linear quantile model in $(1.1)$ is an useful generalization of both classical linear quantile regression model and functional linear quantile regression model. For mean regression models, recent studies (Shin, 2009; Lu et al., 2014) considered the estimation of partially functional linear regression models when the covariates are measured without classical measurement error. Kong et al. (2016) developed a penalized estimation procedure for variable selection in a high-dimensional partially functional linear regression model. In terms of quantiles, Kato (2012) studied the estimation of the model when the covariates are measured without any error. When the functional covariates are measured with error, aforementioned methods can no longer be applicable. Ignoring measurement errors can lead to biased estimation and misleading inference. When the functional covariate is contaminated with error, consistent estimation of the model in $(1.1)$ is technically challenging. Existing methods often require complete specification of the conditional distributions of the response given the true functional covariates, which are often impractical. In addition, most existing methods focus on a fixed number of scalar covariates measured with error, while the model in (1.1) involves a functional covariate of infinite 
dimension, which imposes both computational and theoretical difficulties.

\section{The Proposed Method}

Functional principal component analysis (FPCA) plays an important role in functional data analysis, as it provides an efficient mechanism to represent random functions as a linear combination of basis functions. Our method is based on the FPCA of covariate process $X(t)$. Suppose that $\int_{0}^{1} \mathrm{E}\left[X^{2}(t)\right] d t<$ $\infty$. Denote the covariance kernel of $X(t)$ as $K_{x}(s, t)=\operatorname{Cov}(X(s), X(t))$. Then, the Hilbert-Schmidt theorem entails that $K_{x}(s, t)$ can be represented as $K_{x}(s, t)=\sum_{j=1}^{\infty} \kappa_{j} \phi_{j}(s) \phi_{j}(t)$, where $\kappa_{1} \geq \kappa_{2} \geq \ldots \geq 0$ are ordered eigenvalues, and $\left\{\phi_{j}\right\}_{j=1}^{\infty}$ is an orthonormal basis of $L_{2}[0,1]$. Thus, we have the following expansions in $L_{2}[0,1], X_{i}(t)=\sum_{j=1}^{\infty} X_{i j} \phi_{j}(t), U_{i}(t)=\sum_{j=1}^{\infty} U_{i j} \phi_{j}(t)$, $\beta_{0}(t, \tau)=\sum_{j=1}^{\infty} b_{0 j}(\tau) \phi_{j}(t)$, where $X_{i j}, U_{i j}$ and $b_{0 j}(\tau)$ are defined by $X_{i j}=$ $\int_{0}^{1} X_{i}(t) \phi_{j}(t) d t, U_{i j}=\int_{0}^{1} U_{i}(t) \phi_{j}(t) d t, b_{0 j}(\tau)=\int_{0}^{1} \beta_{0}(t, \tau) \phi_{j}(t) d t$. Thus, model (1.1) can be represented by a linear quantile regression model with an infinite number of "regressors",

$$
Q_{Y_{i} \mid X_{i}, \mathbf{Z}_{i}}(\tau)=\sum_{j=1}^{\infty} b_{0 j}(\tau) X_{i j}+\mathbf{Z}_{i}^{T} \boldsymbol{\theta}_{0}(\tau)
$$

If $X(t)$ is observed without error, a truncated version of 1.3 is often considered, where $\sum_{j=1}^{\infty} b_{0 j}(\tau) X_{i j}$ is truncated by $\sum_{j=1}^{m} b_{0 j}(\tau) X_{i j}$ with a large integer $m$ and 
the unknown coefficients can be estimated by

$$
(\tilde{\mathbf{b}}(\tau), \tilde{\boldsymbol{\theta}}(\tau))=\underset{(\mathbf{b}, \boldsymbol{\theta})}{\arg \min } \sum_{i=1}^{n} \rho_{\tau}\left(Y_{i}-\mathbf{X}_{i}^{T} \mathbf{b}(\tau)-\mathbf{Z}_{i}^{T} \boldsymbol{\theta}(\tau)\right)
$$

where $\rho_{\tau}(\epsilon)=\{\tau-I(\epsilon \leq 0)\} \epsilon$ is the check loss at the $\tau$-th quantile. Similar estimation procedure has been considered in quantile regression involving functional covariates without classic measurement errors. Kato (2012) systematically investigated the asymptotic property of such estimator in a functional linear quantile model. Yao et al. (2017) considered a regularized procedure for simultaneous variable selection and estimation.

In this paper, we consider a scenario where the functional covariate is not fully observed, instead it is contaminated with classic measurement error. It means that measurement error is additive and independent of responses and covariates in the regression model. Our goal is to construct consistent estimators of regression coefficients in (1.1) in the presence of classic measurement error. Applying similar basis expansions on both sides of measurement error equation (1.2), we have, for $j=1, \ldots, m$,

$$
W_{i j}=X_{i j}+U_{i j}
$$

where $W_{i j}=\int_{0}^{1} W_{i}(t) \phi_{j}(t) d t$. 
When $X_{i}(t)$ is contaminated with measurement error, naively replacing $X_{i j} \mathrm{~s}$ with $W_{i j}$ s into the objective function $(1.4)$ can lead to biased and inconsistent estimators of unknown parameters. Instead, we adopt the corrected loss approach proposed in Wang et al. (2012) for linear quantile regression models and extend it to partially functional quantile regression models. Our estimation strategy is based on a corrected objective or loss function which leads to unbiased estimation of a smooth approximation of $\rho_{\tau}\left(Y_{i}-\mathbf{X}_{i}^{T} \mathbf{b}(\tau)-\mathbf{Z}_{i}^{T} \boldsymbol{\theta}(\tau)\right)$. The corrected objective function makes use of a property of Gaussian random variables $($ Stefanski and Cook, 1995$)$ that $\mathrm{E}\left\{\mathrm{E}\left[\phi\left(Z_{1}+\mathrm{i} \sigma Z_{2} \mid Z_{1}\right)\right]\right\}=\phi(\mu)$, where $\mathrm{i}=\sqrt{-1}$, for two independent Gaussian random variables $Z_{1} \sim N\left(\mu, \sigma^{2}\right)$ and $Z_{2} \sim N(0,1)$ as long as $\phi(\cdot)$ is a sufficiently smooth function. Another key element in the construction of the corrected loss function is to use an infinitely smooth function $\rho_{h}(\cdot)$ to approximate the check loss $\rho_{\tau}(\cdot)$ in quantile regression with $\rho_{h}(\epsilon)=\epsilon\left[\tau-1 / 2+\pi^{-1} \int_{0}^{\epsilon / h} \sin (t) / t d t\right]$. Here $h$ controls the goodness of approximation with $\lim _{h \rightarrow 0} \rho_{h}(\epsilon)=\rho_{\tau}(\epsilon)$.

Motivated by Wang et al. (2012), when the measurement errors follow normal distribution, we consider the following corrected quantile objective function,

$$
(\hat{\mathbf{b}}(\tau), \hat{\boldsymbol{\theta}}(\tau))=\operatorname{argmin}_{(\mathbf{b}, \theta)} \sum_{i=1}^{n} \rho_{h}^{*}\left(Y_{i}-\mathbf{W}_{i}^{T} \mathbf{b}(\tau)-\mathbf{Z}_{i}^{T} \boldsymbol{\theta}(\tau), \mathbf{b}^{T} \Sigma_{u} \mathbf{b}\right),
$$


where $\rho_{h}^{*}\left(\epsilon, \sigma^{2}\right)=\pi^{-1} \int_{0}^{1 / h}\left\{y^{-1} \epsilon \sin (y \epsilon)-\sigma^{2} \cos (y \epsilon)\right\} \exp \left(y^{2} \sigma^{2} / 2\right) d y+\epsilon(\tau-$ $1 / 2)$, and $\Sigma_{u}=\operatorname{Cov}\left(\mathbf{U}_{i}\right)$. The function $\rho_{h}^{*}$ is constructed so that it is an unbiased estimator of $\rho_{h}$. In particular, Wang et al. (2012) showed that $\mathrm{E}\left[\rho_{h}^{*}\left(Y-\mathbf{W}^{T} \mathbf{b}(\tau)\right.\right.$ $\left.\left.-\mathbf{Z}^{T} \boldsymbol{\theta}(\tau), \mathbf{b}^{T} \Sigma_{u} \mathbf{b}\right) \mid Y, X, \mathbf{Z}\right]=\rho_{h}\left(Y-\mathbf{X}^{T} \mathbf{b}(\tau)-\mathbf{Z}^{T} \boldsymbol{\theta}(\tau)\right) \approx \rho_{\tau}\left(Y-\mathbf{X}^{T} \mathbf{b}(\tau)\right.$ $\left.-\mathbf{Z}^{T} \boldsymbol{\theta}(\tau)\right)$, and the parameter $h$ controls the goodness of approximation with a better approximation for a smaller $h$. Thus consistent estimators of coefficients $\theta(\tau)$ and $\beta(t, \tau)$ can be obtained from 1.6, , with $\hat{\beta}(t, \tau)=\sum_{j=1}^{m} \hat{b}_{j}(\tau) \hat{\phi}_{j}(t)$ and $\left\{\hat{\phi}_{j}(t)\right\}_{j=1}^{m}$ is orthonormal basis of the estimated $K_{x}(s, t)$ as in subsection 3.1.

\subsection{Implementation}

Notice that $(\hat{\mathbf{b}}(\tau), \hat{\boldsymbol{\theta}}(\tau))$ in 1.6 is not feasible in practice since it relies on $K_{x}(s, t)$, which is generally unavailable when functional covariate $X$ is not fully observed. Therefore additional data are needed for the estimation and identification of $K_{x}(s, t)$. In this paper, we use repeated observations to estimate $K_{x}(s, t)$ and $K_{u}(s, t)$ due to measurement errors. Suppose we have $R$ repeated observations of $W_{i}(t)$ denoted as $W_{i}^{r}(t)$ for $r=1, \ldots, R$ and $i=1, \ldots, n$. After simple interpolation rule as in Kato (2012), $K_{x}(s, t)$ is estimated as $\hat{K}_{x}(s, t)=\hat{K}_{w}(s, t)-\hat{K}_{u}(s, t)$, where $\hat{K}_{w}(s, t)=\sum_{i=1}^{n}\left[W_{i}(s)-\right.$ $\bar{W}(s)]\left[W_{i}(t)-\bar{W}(t)\right] /(n-1), \hat{K}_{u}(s, t)=\sum_{i=1}^{n} \sum_{r=1}^{R}\left[W_{i}^{r}(s)-\bar{W}_{i}(s)\right]\left[W_{i}^{r}(t)-\right.$ $\left.\bar{W}_{i}(t)\right] /(n R-n)$, with $\bar{W}_{i}(t)=\frac{1}{R} \sum_{r=1}^{R} W_{i}^{r}(t)$ and $\bar{W}(t)=\frac{1}{n} \sum_{i} W_{i}(t)$. Let the empirical eigen-decomposition of $\hat{K}_{x}(s, t)$ be $\hat{K}_{x}(s, t)=\sum_{j=1}^{m} \hat{\kappa}_{x j} \hat{\phi}_{j}(s) \hat{\phi}_{j}(t)$. 
Let $\hat{W}_{i j}=\int_{0}^{1} W_{i}(t) \hat{\phi}_{j}(t) d t$ and $\hat{W}_{i j}^{r}=\int_{0}^{1} W_{i}^{r}(t) \hat{\phi}_{j}(t) d t$ be the empirical projections of $W_{i}(t)$ and $W_{i}^{r}(t)$ on to $\hat{\phi}_{j}(t)$ respectively for $j=1, \ldots, m$. Furthermore, let $\hat{\mathbf{W}}_{i}^{r}=\left(\hat{W}_{i 1}^{r}, \cdots, \hat{W}_{i m}^{r}\right)^{T}$ be the $m$ dimensional vector composed of empirical components for $W_{i}^{r}(t)$. Then $\Sigma_{u}$ can be estimated using the within subject covariance of the repeated empirical components $\hat{\mathbf{W}}_{i}^{r}$. Specifically, $\hat{\Sigma}_{u}=$ $\sum_{i=1}^{n} \sum_{r=1}^{R}\left(\hat{\mathbf{W}}_{i}^{r}-\overline{\mathbf{W}}_{i}^{*}\right)\left(\hat{\mathbf{W}}_{i}^{r}-\overline{\mathbf{W}}_{i}^{*}\right)^{T} /(n R-n)$ with $\overline{\mathbf{W}}_{i}^{*}=\frac{1}{R} \sum_{r=1}^{R} \hat{\mathbf{W}}_{i}^{r}$. Now we can obtain our estimators by

$$
(\check{\mathbf{b}}(\tau), \check{\boldsymbol{\theta}}(\tau))=\operatorname{argmin} \sum_{i=1}^{n} \rho_{h}^{*}\left(Y_{i}-\hat{\mathbf{W}}_{i}^{T} \mathbf{b}(\tau)-\mathbf{Z}_{i}^{T} \boldsymbol{\theta}(\tau), \mathbf{b}^{T} \hat{\Sigma}_{u} \mathbf{b}, h\right)
$$

and the functional coefficient is estimated as $\check{\beta}(t, \tau)=\sum_{j=1}^{m} \check{b}_{j}(\tau) \hat{\phi}_{j}(t)$.

Another practical issue is to determine $m$, the number of eigen functions, and tuning parameter $h$. In the simulation, we use the Bayesian information criterion (BIC) to determine $m$, which is more stable as suggested in (Kato, 2012). When there is no measurement error, the oracle estimation gives $B I C(m)=$ $\log \left[\frac{1}{n} \sum_{i=1}^{n} \rho_{\tau}\left(Y_{i}-Z_{i}^{T} \hat{\theta}(\tau)-\sum_{j=1}^{m} \hat{b}_{j}(\tau) \hat{X}_{i j}\right)\right]+(m+p) \log (n) / n$. The BIC can be defined similarly for the naive method by replacing $\hat{X}_{i j}$ with $\hat{W}_{i j}$. As shown in Table 1 in the supplementary material, the number of selected scores using naive and oracle methods are close. Thus we propose to use naive method to choose $m$, and the same $m$ is also used for the proposed corrected loss method. 
As for the tuning parameter $h$ in corrected quantile objective function, we apply the simulation and extrapolation method (SIMEX) as in Wang et al. (2012) and Delaigle and Hall (2008). For a given $m$, let $\hat{\gamma}(h)=\left(\hat{\mathbf{b}}^{T}(h), \hat{\theta}(h)\right)$ be the corrected loss estimator associated with $h$. Here we omit $\tau$ for notation simplicity. An optimal $h_{0}$ is the one that minimizes the mean squared error of $\hat{\gamma}(h)$, defined as $\mathrm{E}\left[\left(\hat{\gamma}(h)-\gamma_{0}\right)^{T} \Sigma_{\hat{\gamma}}^{-1}\left(\hat{\gamma}(h)-\gamma_{0}\right)\right]$, where $\gamma_{0}$ is the true regression coefficients and $\Sigma_{\hat{\gamma}}$ is the covariance matrix of $\hat{\gamma}(h)$. Since the mean squared error depends on unknown covariate $X(t)$, it can not be directly calculated. Instead we estimate it using the simulation and extrapolation method. In the simulation step, we generate additional independent error terms $\left\{\mathbf{U}_{i, 1}\right\}_{i=1}^{n}$ and $\left\{\mathbf{U}_{i, 2}\right\}_{i=1}^{n}$ from $\mathbf{N}\left(\mathbf{0}, \hat{\Sigma}_{u}\right)$ and obtain new surrogate variables $\left\{\mathbf{W}_{i, 1}^{*}=\mathbf{W}_{i}+\mathbf{U}_{i, 1}\right\}_{i=1}^{n}$ and $\left\{\mathbf{W}_{i, 2}^{*}=\mathbf{W}_{i, 1}^{*}+\mathbf{U}_{i, 2}\right\}_{i=1}^{n}$ with increasing levels of measurement error. Based on the two simulated data sets $\left\{Y_{i}, \mathbf{Z}_{i}, \mathbf{W}_{i, 1}^{*}\right\}_{i=1}^{n}$ and $\left\{Y_{i}, \mathbf{Z}_{i}, \mathbf{W}_{i, 2}^{*}\right\}_{i=1}^{n}$, we obtain the corrected loss estimators $\hat{\gamma}_{1}(h)$ and $\hat{\gamma}_{2}(h)$ respectively. Repeat the simulation step for $N_{s}$ times. Through $\left\{\hat{\gamma}_{1, s}(h)\right\}_{s=1}^{N_{s}}$ and $\left\{\hat{\gamma}_{2, s}(h)\right\}_{s=1}^{N_{s}}$, we can estimate the mean squared error of $\hat{\gamma}_{1}(h)$ and $\hat{\gamma}_{2}(h)$ respectively as

$$
\begin{aligned}
& M_{1}(h)=N_{s}^{-1} \sum_{s=1}^{N_{s}}\left[\hat{\gamma}_{1, s}(h)-\hat{\gamma}(h)\right]^{T} \hat{\Sigma}_{\hat{\gamma}_{1}}^{-1}\left[\hat{\gamma}_{1, s}(h)-\hat{\gamma}(h)\right], \\
& M_{2}(h)=N_{s}^{-1} \sum_{s=1}^{N_{s}}\left[\hat{\gamma}_{2, s}(h)-\hat{\gamma}_{1, s}(h)\right]^{T} \hat{\Sigma}_{\hat{\gamma}_{2}}^{-1}\left[\hat{\gamma}_{2, s}(h)-\hat{\gamma}_{1, s}(h)\right]
\end{aligned}
$$


where $\hat{\Sigma}_{\hat{\gamma}_{1}}$ and $\hat{\Sigma}_{\hat{\gamma}_{2}}$ are the sample covariance matrices of $\left\{\hat{\gamma}_{1, s}(h)-\hat{\gamma}(h)\right\}_{s=1}^{N_{s}}$ and $\left\{\hat{\gamma}_{2, s}(h)-\hat{\gamma}_{1, s}(h)\right\}_{s=1}^{N_{s}}$ respectively. Let $\hat{h}_{1}=\arg \min _{h} M_{1}(h)$ and $\hat{h}_{2}=$ $\arg \min _{h} M_{2}(h)$. For the extrapolation step, notice that $\left\{\mathbf{W}_{i, 2}\right\}_{i=1}^{n}$ measures $\left\{\mathbf{W}_{i, 1}\right\}_{i=1}^{n}$ in the same way that $\left\{\mathbf{W}_{i, 1}\right\}_{i=1}^{n}$ measures $\left\{\mathbf{W}_{i}\right\}_{i=1}^{n}$, and $\left\{\mathbf{W}_{i}\right\}_{i=1}^{n}$ measures $\left\{\mathbf{X}_{i}\right\}_{i=1}^{n}$. Therefore the relationship between $\hat{h}_{1}$ to $\hat{h}_{2}$ is similar to that between $\hat{h}_{0}$ to $\hat{h}_{1}$. Here $\hat{h}_{0}=\hat{h}$ is the optimal tuning parameter under observed surrogate $\left\{W_{i}\right\}_{i=1}^{n}$. In particular, Delaigle and Hall (2008) considered a linear back-extrapolation with $\log \left(\hat{h}_{1}\right)-\log \left(\hat{h}_{2}\right) \approx \log \left(\hat{h}_{0}\right)-\log \left(\hat{h}_{1}\right)$. Therefor $h_{0}$ can be approximated by $\hat{h}=\hat{h}_{1}^{2} / \hat{h}_{2}$.

\section{Theoretical properties}

We first introduce the following notations. For any $z \in R^{p}$, let $\|z\|$ and $\|z\|_{\infty}$ be the vector $L_{2}$ and supremum norm of $z$ respectively. For any $K:[0,1]^{2} \rightarrow R$, let $\|K\|^{2}=\int_{0}^{1} \int_{0}^{1} K^{2}(s, t) d s d t$. For any two positive sequences $r_{n}$ and $s_{n}$, $r_{n} \asymp s_{n}$ denotes that $r_{n} / s_{n}$ is bounded away from zero and infinity. In addition, $\mathrm{E}_{n}$ denotes the sample mean operator. Moreover, we denote by the same letters $c, C$ for any positive constants, without distinction in each case. To establish the asymptotic results, we need the following assumptions.

Let $K_{x}(s, t)$ be the covariance kernel of $X(t)$ with $\left\{\kappa_{j}\right\}_{j=1}^{\infty}$ and $\left\{\phi_{j}\right\}_{j=1}^{\infty}$ be the eigenvalue and eigen function sequences. Then $\left\{\phi_{j}\right\}_{j=1}^{\infty}$ forms an orthonormal basis of $L_{2}([0,1])$. For $j \geq 1$, let $X_{j}=\int X(t) \phi_{j}(t) d t$ be the projection 
scores. Similarly, define $\left\{\kappa_{u j}\right\}_{j=1}^{\infty},\left\{\phi_{u j}\right\}_{j=1}^{\infty}$ and $\left\{U_{u j}\right\}_{j=1}^{\infty}$ for $U(t)$. To establish the asymptotic results, we need the following assumptions.

(A1) We assume $\left\{Y_{i}, X_{i}(t), Z_{i}, U_{i}(t)\right\}_{i=1}^{n}$ are i.i.d. copies of $\{Y, X(t), Z, U(t)\}$, in which the functional measurement error $\{U(t)\}$ is a zero-mean Gaussian process on $[0,1]$ and independent of $\{Y, X(t), Z\}$.

(A2) The functional covariate satisfies $\int_{0}^{1} \mathrm{E}\left[X^{4}(t)\right] d t \leq c$ and $\mathrm{E}\left[X_{j}^{4}\right] \leq c \kappa_{j}^{2}$, where $c^{-1} j^{-\alpha_{x}} \leq \kappa_{j} \leq c j^{-\alpha_{x}}$ and $\kappa_{j}-\kappa_{j+1} \geq c^{-1} j^{-\alpha_{x}-1}$ for some $\alpha_{x}>1$ and all $j \geq 1$. Similarly, assume $\int_{0}^{1} \mathrm{E}\left[U^{4}(t)\right] d t \leq c$, and $\mathrm{E}\left[U_{u j}^{4}\right] \leq c \kappa_{u j}^{2}$ with $c^{-1} j^{-\alpha_{u}} \leq \kappa_{u j} \leq c j^{-\alpha_{u}}$ for some $\alpha_{u}>1$ and all $j \geq 1$.

(A3) There exist constants $\nu_{1}, \nu_{2} \in(0,2]$ such that $\mathrm{E}\left[(X(t)-X(s))^{2}\right] \leq c \mid t-$ $\left.s\right|^{\nu_{1}}$ and $\mathrm{E}\left[(U(t)-U(s))^{2}\right] \leq c|t-s|^{\nu_{2}}$ for all $s, t \in[0,1]$.

(A4) Let $\mathbf{A}=\left(\tilde{\mathbf{X}}^{T}, \mathbf{Z}^{T}\right)$, where $\tilde{X}_{j}=\kappa_{j}^{-1 / 2} X_{j}$ for each $j$. Assume $c^{-1} \leq$ $\lambda_{\min }\left(E\left(\mathbf{A}^{T} \mathbf{A}\right)\right) \leq \lambda_{\max }\left(E\left(\mathbf{A}^{T} \mathbf{A}\right)\right) \leq c$ for all $\mathrm{n}$, where $\lambda_{\max }$ and $\lambda_{\max }$ are the smallest and largest eigenvalues.

(A5) For some $\beta>\alpha_{x} / 2+1, \sup _{\tau \in(0,1)}\left|b_{0 j}(\tau)\right| \leq c j^{-\beta}$ for all $j \geq 1$.

(A6) For $i=1, \ldots, n$, the functional curve is observed only at discrete points $0=t_{i 1} \leq t_{i 2} \ldots \leq t_{i, L_{i}+1}=1$. Define $\Delta_{n}=\max _{1 \leq i \leq n} \max _{1 \leq l \leq L_{i}}\left(t_{i, l+1}-\right.$ $\left.t_{i l}\right)$. Assume $\Delta_{n} \rightarrow 0, n m^{2 \alpha_{x}} \Delta_{n}^{\nu_{0}}=O(1)$ as $n \rightarrow \infty$, where $\nu_{0}=$ 
$\min \left(\nu_{1}, \nu_{2}\right)$.

(A7) The vector $\theta_{0}$ is an interior point of the parameter space $\Theta$, which is a compact subset of $R^{p}$.

(A8) Let $\varepsilon_{0}(\tau)=Y-Q_{\tau}(Y \mid X(t), Z)$. The conditional density $f\left(\varepsilon_{0}(\tau) \mid X(t), Z\right)$ is continuously differentiable and bounded away from 0 almost surely. In addition, $\mathrm{E}\left(\varepsilon_{0}^{4}(\tau) \mid X(t), Z\right)$ is bounded as a function of $\tau$.

Assumptions on response and functional covariate, similar to (A1)-(A3), (A5)-(A6) and (A8), can also be found in Kato (2012), which are needed to establish the estimation consistency of the functional coefficient function when there is no measurement error. In particular, (A2)-(A3) determine the smoothness of random functions $X(t), U(t)$, while (A5) controls the smoothness of the regression function. Similar assumptions can also be found in Hall and Horowitz (2007). (A6) implies that the sampling points are dense in $[0,1]$ as sample size increases. The positive definite matrix assumption in (A4) is similar to Kong et al. (2016). For identifiability purpose, we assume the measurement error is a Gaussian process. The upper bound for $\left\{\mathrm{E}\left[\left(U_{u j}\right)^{4}\right]\right\}_{j=1}^{\infty}$ in assumption (A3) is a sufficient condition satisfying for bounded moments of $\left\{U_{j}\right\}_{j=1}^{\infty}$.

Theorem 1. Under assumptions (Al)-(A8), if $m^{2 \alpha_{x}+2} / n \rightarrow 0$, then as $n \rightarrow \infty$ 
and $h \rightarrow 0$,

$$
\begin{aligned}
\int_{0}^{1}\left\{\hat{\beta}(t, \tau)-\beta_{0}(t, \tau)\right\}^{2} d t & =O_{p}\left[\frac{h^{-1} \exp \left(c h^{-2}\right) m^{\alpha_{x}+1 / 2}[\log (m+n)]^{1 / 2}}{n^{1 / 2}}+h m^{\alpha_{x}}\right] \\
& +O_{p}\left(m n^{-1}\right)+O_{p}\left(m^{-2 \beta+1}\right) .
\end{aligned}
$$

Remark 1. Theorem 1 shows that our functional estimator is consistent in probability. The rate of convergence is composed of three terms, which arise from the truncation and estimation errors associated with the approximation of functional coefficient $\beta_{0}(t, \tau)$ with orthonormal basis $\left\{\phi_{j}\right\}_{j=1}^{m}$, and the approximation of the check function in quantile regression with a smooth differentiable function to incorporate measurement errors. Often the second term is dominated by the third term for some $m$, of which $m \asymp n^{1 /\left(\alpha_{x}+2 \beta\right)}$ in Kato (2012) is such an example. Let $h=c(\log n)^{-\delta}$ and $m=c(\log n)^{\delta /\left(2 \beta+\alpha_{x}-1\right)}$ for some $0<\delta<1 / 2$. Then the rate of convergence simply reduces to $O_{p}\left(m^{-2 \beta+1}\right)$. This rate of convergence can also be found in Kato (2012), while it requires higher order of number of eigen functions with $m \asymp n^{1 /\left(\alpha_{x}+2 \beta\right)}$.

To establish asymptotic normality of $\hat{\boldsymbol{\theta}}$, we require additional assumptions.

(A9) For $l=1, \ldots, p$, there exists $g_{l}(t)$ such that $Z_{l}^{*}=Z_{l}-\mathrm{E}\left(Z_{l}\right)-\int_{0}^{1} X(t) g_{l}(t) d t$ satisfying $\mathrm{E}\left[Z_{l}^{*} \mid X(t)\right]=0$ and $\left|g_{l j}\right| \leq c j^{-\beta}$ with $g_{l j}=\int_{0}^{1} g_{l}(t) \phi_{j}(t) d t$ for each $l$ and $j \geq 1$. In addition, denote $\tilde{Z}_{l}=Z_{l}-\mathrm{E}\left(Z_{l}\right)-\int_{0}^{1} W(t) g_{l}(t) d t$. 
(A10) Denote $\mathbf{Z}^{*}=\left(Z_{1}^{*}, \ldots, Z_{p}^{*}\right)^{T}$ and $\tilde{\mathbf{Z}}=\left(\tilde{Z}_{1}, \ldots, \tilde{Z}_{p}\right)^{T}$. There exist positive definite matrices $\mathbf{B}$ and $\mathbf{D}$ such that $\mathrm{E}\left\{\tilde{\mathbf{Z}} \tilde{\mathbf{Z}}^{T}\left[\frac{\partial \rho_{h}^{*}}{\partial \varepsilon}\left(\varepsilon_{0}-\mathbf{U}^{T} \mathbf{b}_{0}, \mathbf{b}_{0}^{T} \Sigma_{u} \mathbf{b}_{0}\right)\right]^{2}\right\} \rightarrow$ $\mathbf{B}$ and $\mathrm{E}\left[\mathbf{Z}^{*} \mathbf{Z}^{* T} \frac{\partial^{2} \rho_{h}^{*}}{\partial \varepsilon^{2}}\left(\varepsilon_{0}-\mathbf{U}^{T} \mathbf{b}_{0}, \mathbf{b}_{0}^{T} \Sigma_{u} \mathbf{b}_{0}\right)\right] \rightarrow \mathbf{D}$, as $n \rightarrow \infty$ and $h \rightarrow$ 0 . Furthermore, we assume $\frac{\partial^{2} \rho_{h}^{*}}{\partial \varepsilon \partial \sigma^{2}}\left(\varepsilon_{0}-\mathbf{U}^{T} \mathbf{b}_{0}, \mathbf{b}_{0}^{T} \Sigma_{u} \mathbf{b}_{0}\right)$ and $\left[\frac{\partial^{2} \rho_{h}}{\partial \varepsilon^{2}}\left(\varepsilon_{0}\right)\right]^{2}$ have bounded expectations conditional on $\mathbf{Z}$ and $X(t)$.

Remark 2. Assumptions similar to (A9) can be found in Lu et al. (2014) and Shin (2009), which is introduced to adjust the dependence between $\mathbf{Z}$ and $X(t)$. The first part of assumption (A10) is exactly the same as assumption 7 in Wang et al. (2012) in the case of error-free covariates. While bounded expectation of the second order derivatives is need for the partially linear functional model.

Theorem 2. Under the same assumptions as Theorem 1 and Assumptions (A9) and (A10), we have $\sqrt{n}\left(\hat{\boldsymbol{\theta}}-\boldsymbol{\theta}_{0}\right) \rightarrow N\left(\mathbf{0}, \mathbf{D}^{-\mathbf{1}} \mathbf{B D}^{-\mathbf{1}}\right)$ in distribution.

\section{Simulation studies}

In this section, we conduct simulation studies to assess the finite sample performance of the proposed corrected loss method (CL), and compare it with the naive method (NAIVE) which ignores measurement errors by simply replacing $X_{i j}$ with $W_{i j}$ in (1.4). As a benchmark, we also consider the oracle estimator (ORACLE) assuming the functional covariate $X(t)$ is fully observed. Data are independently generated from the model $Y=\int_{0}^{1} b(t) X(t)+\left(Z_{1}, Z_{2}\right) \boldsymbol{\theta}+\epsilon$, and $W(t)=X(t)+U(t)$, where $b(t)=\sum_{j=1}^{50} b_{j} \phi_{j}(t)$, with $b_{1}=0.3, b_{j}=$ 
$5(-1)^{j+1} / j^{3.5}$ for $j \geq 2, X(t)=\sum_{j=1}^{50} \gamma_{j} Z_{j} \phi_{j}(t)$ with $\gamma_{j}=(-1)^{j+1} j^{-\alpha / 2}$,

$Z_{j} \sim U[-\sqrt{3}, \sqrt{3}]$, and $U(t)=\sum_{j=1}^{50} \nu_{j} U_{j} \phi_{j}(t)$ with $\nu_{j}=(-1)^{j+1} j^{-\alpha / 2}$,

$U_{j} \sim N(0,1)$, and the regression error $\epsilon=\left\{1+\eta \int_{0}^{1} \cos (\pi t) X(t) d t\right\} N(0,1)$.

We consider two different values $\eta=0$ and 0.5 in Case 1 and Case 2 respectively, corresponding to homogeneous and heteroscedastic regression models respectively. In Case 3, $\epsilon$ is generated from a $t$ distribution with 5 degrees of freedom, but is normalized to have mean zero and variance 1 . We consider basis functions $\phi_{j}(t)=\sqrt{2} \cos (\pi j t)$ and the parameter $\alpha=1.1$ or 2 , which controls the effective number of basis functions needed for functional data. For the parametric part, we consider the regression coefficients $\boldsymbol{\theta}=(0.3,0.5)^{T}$, and covariates $Z_{1} \sim \operatorname{Binomial}(1,0.6)$, and $Z_{2} \sim N(\mu, 0.25)$, where $\mu$ depends on the functional covariate $X(t)$ by $\mu=1+\int_{0}^{1} \sum_{j=1}^{5}(-1)^{j+1} \frac{1}{j^{2}} \phi_{j}(t) X(t) d t$. We set $n=200,400,600$ and repeat the experiment 100 times. In the following, we consider the proposed estimation method at both 50th and 75th quantiles.

For our method, we assume $X(t)$ is unobserved. Instead several replications of $W(t)$ are observed to estimate covariance function $K_{U}(s, t)$ of the measurement error. Here we estimate $K_{U}(s, t)$ based on three replications of $W(t)$ and the average of three replications is taken as observed $W(t)$. Then for any two time points $s$ and $t$, the covariance function of $X(t)$ can be estimated as $\hat{K}_{X}(s, t)=\hat{K}_{W}(s, t)-\hat{K}_{U}(s, t)$. We then calculate $\left\{\hat{\phi}_{j}(t)\right\}_{j=1}^{m}$ as the first $m$ 
eigen functions of $\hat{K}_{X}(s, t)$. The number of eigen functions $m$ is selected using the BIC in subsection 3.1. For the selection of $h$, the SIMEX method in subsection 3.1 is used with $N_{s}=20$ in the simulation step. To save computational time, SIMEX selection is performed on only 30 experiments, and the average of 30 values $\hat{h}_{\text {simex }}$ is used for each of the 100 replications. The average number of selected eigen functions with standard deviations and the tuning parameter $h$ are reported in Tables 1 and 2 of the supplementary material respectively.

Figure 1 presents boxplots of $\hat{\theta}_{k}(\tau)-\theta_{k}(\tau)(k=0,1,2)$ and Table 1 summarizes the MSE values of $\hat{\theta}_{k}(\tau)(k=0,1,2)$ at $\tau=0.75$ from three different methods. The data are generated with regression error in Case 1 and $\alpha=1.1$ or 2 . Table 1 shows that the CL, NAIVE and ORACLE approaches all give similar estimation results for $\theta_{1}$ with negligible bias under all scenarios due to the fact that $Z_{1}$ is independent with the functional covariate that is contaminated with error. However, since the distribution of $Z_{2}$ is affected by measurement errors, the naive estimates of $\theta_{0}$ and $\theta_{2}$ have obvious biases. In contrast, our method is effective in reducing the estimation bias and results in much smaller biases than the NAIVE method. In terms of estimation variance, the NAIVE approach gives the smallest variances because of extra variability in the observed covarites due to measurement errors. However, Table 1 shows that the CL approach has a better overall performance with smaller MSE than the NAIVE method when the sample size 
is larger ( $n=400,600)$, and the overall performance of CL is comparable to the ORACLE approach. In addition, the MSEs for both CL and ORACLE decrease as $n$ increases, supporting our asymptotic results. However, for $\hat{\theta}_{0}$ and $\hat{\theta}_{2}$, the NAIVE method has non-diminishing MSEs due to non-ignorable bias.

The integrated mean squared error (IMSE) is used to assess the accuracy of functional coefficient estimators. Let $\hat{b}_{l}(t, \tau)$ be an estimated coefficient from the $l$-th experiment for $l=1, \ldots, 100$. Let $\bar{b}(t, \tau)=\sum_{l=1}^{100} \hat{b}_{l}(t, \tau) / 100$. We define $\operatorname{Bias}^{2}=\int_{0}^{1}[\bar{b}(t, \tau)-b(t, \tau)]^{2} d t, \operatorname{Var}=\int_{0}^{1} \frac{1}{100} \sum_{l=1}^{100}\left[\hat{b}_{l}(t, \tau)-\bar{b}(t, \tau)\right]^{2} d t$, and IMSE $=\int_{0}^{1} \frac{1}{100} \sum_{l=1}^{100}\left[\hat{b}_{l}(t, \tau)-b(t, \tau)\right]^{2} d t$

Table 2 reports Bias $^{2}$, Var and IMSE of three functional coefficient estimates. It again shows the effectiveness of the proposed method in reducing estimation bias due to measurement error. The size of the $\mathrm{Bias}^{2}$ under the CL is smaller than the NAIVE approach, and close to the ORACLE estimator in particular when $n$ is large. For larger $n$, our proposed method obtains smaller values of IMSE than the naive method. In addition, when $\alpha$ increases from 1.1 to 2 , the IMSE of $\hat{b}(t, \tau)$ is larger which is consistent with what implied by Remark 1. The same phenomenon was also observed in Kato (2012). Figure 2 plots the average of functional coefficient estimates over 100 replications for each of three different methods. The curves of the oracle estimator and our proposed estimator are closer to the true line than the naive curve at each $\alpha$ and sample 
size $n$. Other results which are not provided here show the same conclusions.

To investigate the performance of the proposed estimator under different regression error distributions, we generate data from the above model with $n=$ 400 and $\alpha=1.1$, but with three different regression error distributions as described in cases 1,2 and 3. We also compare the performance of the proposed method at both 50-th and 75-th quantiles. Tables 3 and 4 in the supplementary material report the estimation results. Under all error distributions and both quantile levels, the NAIVE gives non-ignorable biases in estimating both parametric and nonparametric regression coefficients. As a contrast, the proposed method is effective in reducing estimation biases under all scenarios.

We also use simulation to verify the asymptotic variance formula for the parametric part given in Theorem 2. Data of sizes $n=200,400,600$ are generated from Case 1 with $\tau=0.5$ and $\alpha=2$. Table 3 compares the empirical standard errors with the theoretical ones calculated using the asymptotic formula in Theorem 2 for $\hat{\theta}_{0}, \hat{\theta}_{1}$, and $\hat{\theta}_{2}$ respectively. It shows that the empirical and the theoretical standard errors are close for all three parametric regression coefficients, thus supporting asymptotic results in Theorem 2 .

However, it is challenging to use the theoretical standard errors to make inference on the regression coefficients. The calculation of the theoretical standard errors relies on the true data generating process, which is unavailable and needs 
to be estimated in practice. In addition, it involves the projection of error free covariates on $\{X(t)\}$, which is not feasible in practice since $\{X(t)\}$ is unobserved due to measurement error. Therefore, we propose to use bootstrap method to construct confidence intervals for the unknown regression coefficients. Table 4 provides empirical coverage probabilities of $95 \%$ confidence intervals based on 500 bootstrap samples for $\tau=0.5, \alpha=2$ of Case 1. For the functional coefficient, the coverage probability of the pointwise confidence interval is calculated by averaging the coverage probabilities at 201 observed time points in the interval of $[0,1]$. Table 4 shows that the bootstrap approach performs reasonably well with the empirical coverage probabilities close to $95 \%$.

\section{Real data analysis}

In this section, we apply the proposed method for childhood obesity study. About $20 \%$ of children in the U.S. are obese, and the prevalence of childhood obesity has more than tripled in the last 40 years. Childhood obesity negatively affects physiological, behavioral, and psychological developments. To combat the obesity epidemic, researchers have begun implementing behavioral schoolbased interventions for increasing physical activity in children. In this section, we apply our proposed method to the data set from a children obesity study conducted by Dr. Mark Benden and colleagues from 2012 to 2014.

In this study, a total of 230 students from three different elementary schools 
in the College Station Independent School District were enrolled and followed over a 6-month period. The students were randomly assigned to receive either stand-biased desks (treatment) or traditional desks (control) in classroom. The purpose of the study is to evaluate the effect of stand-biased desks as an intervention for increasing energy expenditure and reducing obesity in elementary school-aged children. In our application, daily energy expenditure, $X(t)$, is defined as the total number of calories or energy used by the body to perform every-day bodily functions. The true values of $X(t)$ are not directly observable. Instead the surrogate measure for daily energy expenditure, $W(t)$, was collected per minute from the Sense Wear Armband ${ }^{\circledR}$ (BodyMedia, Pittsburgh, PA) for students who wore accelerometers while in school for one week (5 days) at baseline. When energy expenditure measurements have missing values, cubic splines are applied to smooth each individual energy expenditure curve, which is then used to impute the missing values. In addition to accelerometry-based energy expenditure data, other covariates collected at baseline include each subject's school, age, sex, ethnicity, height, and treatment. The covariate treatment is a binary variable used to indicate desk assignment with value 1 for stand-biased desks and 0 for traditional desk. BMI values are measured both at baseline and after 6 months for all 230 students in the study. We take the average of two BMI values to reflect the overall BMI level during the 6 month period for each 
student. Table 5 provides a summary of the variables.

Students from different schools enrolled in the study at different time points. To eliminate any potential effect of enrollment time on students' daily energy expenditure patterns, we center the measured energy expenditure data by subtracting the daily averaged energy expenditure from each individual curve. In addition, the daily energy expenditure curve is averaged for every 5 minutes to reduce the variability, which leaves 52 data points in each curve. Also, the time interval is scaled to $[0,1]$. Since some students only have three days' measurements of energy expenditure, we randomly pick three days' data to estimate the covariance kernel of $X(t)$ and variance of measurement errors. Figure 3 provides the plots of three days' data where $W_{1}(t), W_{2}(t)$ and $W_{3}(t)$ are plotted against time for all subjects. The grey lines are individual energy expenditure observations while the black solid line is the mean energy expenditure.

We consider the following models for $Q_{\tau}$, the $\tau$-th conditional quantile of $\log (\mathrm{BMI})$ and measurement errors $Q_{\tau}=\theta_{0}+\theta_{1} S_{1}+\theta_{2} S_{2}+\theta_{3} \operatorname{Tr} t+\theta_{4}$ Sex + $\theta_{5} R_{1}+\theta_{6} R_{2}+\theta_{7} A g e+\int X(t) \beta(t) d t$, and $W_{j}(t)=X(t)+U_{j}(t)$, for $j=1,2,3$, where $S_{1}$ and $S_{2}$ are binary indicators for School 1 and School 2 respectively, and School 3 is set as the baseline, Trt is a binary treatment indicator for desk assignment, and Sex is binary variable taking value 1 for male and 0 for female students. For ethnicity, $R_{1}$ and $R_{2}$ are binary indicators for Other Ethnicity and 
Blacks respectively while White is the reference category. For illustration, we consider $\tau=0.2,0.5,0.8$ and compare our proposed method with the naive method, which ignores measurement errors. The number of basis used to represent the functional data is selected by BIC criterion based on the naive method. It selected one basis $(m=1)$ for all three levels of $\tau$. By SIMEX, we choose $h$ as $2.116,3.462,1.900$ at $\tau=0.2,0.5,0.8$ respectively for our method.

The estimated functional coefficients are provided in Figure 4. For each quantile level, the $95 \%$ nonparametric bootstrap confidence intervals do not contain the zero line, indicating that energy expenditure has a significant effect on the 20th, 50th, 80th quantiles of $\log (\mathrm{BMI})$ respectively. In addition, the estimated coefficient functions are positive indicating more daily energy expenditures associated with higher BMI values. This may be due to the fact that higher energy cost is needed to perform weight-bearing activities for individuals with higher BMI values. This positive association was also observed in Maffeis et al. (1996). In addition, Figure 4 shows that the naive estimates attenuate its effects toward zero compared with the proposed estimates. Table 6 reports the estimation results of parametric regression coefficients. As expected, the estimates of the interceptor increase with quanitle level $\tau$ and all are significantly different from zero. Our proposed estimates indicates significant school effects at 50th and 80th quantiles, while no significant school effect is found at 20th quanitle 
due to larger variability of the proposed method. For desk assignment (trt), there is no significance finding at all quantile levels for both methods. This is possibly due to its effect is mediated by the inclusion of the energy expenditure in the model. For Sex, our proposed estimates show significant effects at the 50th quantile rather than at the 80th quantile indicated by the naive estimates. Both methods reveal no significant effects of ethnicity on $\log (\mathrm{BMI})$ at three quantile levels. For age, both methods find significant positive effect for median BMI. However, the proposed method finds age to be signficant at 80th quanitle, while naive method found it to be signficiant at 20th quantile.

\section{Discussion}

In this paper we establish consistent estimators for partially functional linear quantile model when the functional covariate is contaminated with functionvalued error. The non-differentiable check loss in quantile regression and functional measurement error impose additional challenges. Our method neither requires specification of the conditional distribution of the response given the true covariates nor assumes independence between measurement errors at different time points. We assume the functional measurement error is a Gaussian process and develop the corrected loss function based on a smooth function of check loss function, where a smoothing parameter needs to be determined for a bias and variance trade-off. We show the consistency and asymptotic normality of 
proposed functional estimator and parametric estimator respectively. Simulations and real data analysis indicate our method outperforms the naive method.

The proposed model only allows for one functional predictor. But it can be extended to allow multiple functional predictors. With the basis representation, it leads to more terms in either $\mathbf{Z}$ and/or $\mathbf{X}$ depending on whether the functional predictors are measured with or without errors. While the algorithm proposed in this paper can be extended to obtain resulting estimators, the proofs for establishing asymptotic results require additional work due to extra functional predictors.

In implementation, we determine the number of eigen functions by simply ignoring the existence of measurement errors. A more precise method should be investigated to determine the number of eigen functions in the presence of measurement errors. Furthermore, we could consider more general measurement error model, e.g., the multiplicative measurement error or relaxing the Gaussian assumption of measurement error which is used in the present method.

In addition, Laplace distribution is another popular distribution for the measurement errors. In fact, Wang et al. (2012) considered the corrected score approach for measurement error models with Laplace distribution in linear quantile regression. However, one challenge to extend it to the functional covariate case is that Laplace distribution does not have the linear additive property. That is, a linear combination of Laplace random variables does not necessarily fol- 
low the Laplace distribution. Therefore, if the functional measurement errors are assumed to follow multivariate Laplace distribution, the individual scores no longer follow the Laplace distribution. Thus, the extension to Laplace functional measurement errors is not straightforward, and more investigation is needed.

\section{Supplementary Material}

The Supplementary Material includes additional simulation results, detailed proofs of the main theorems and necessary lemmas.

\section{Acknowledgements}

The authors thank Mark Benden for providing the data used in the application section. The authors also would like to thank the reviewers, the associate editor and the co-editor for their helpful suggestions and comments. Xue's research was supported by the Simons Foundation (F0782A) and National Science Foundation (DMS-1812258). Tekwe's research was supported by National Cancer Institute Supplemental Award U01-CA057030-29S2. Bai's research was supported by Natural Science Foundation of China (11771268). Qu's research was supported by the National Science Foundation (DMS-1821198). 
PARTIALLY FUNCTIONAL LINEAR QUANTILE REGRESSION

Table 1: MSEs of parametric coefficient estimators from proposed method (CL), the naive method (NAIVE), and the oracle method (ORALCE) at $\tau=0.75$ in Case 1.

\begin{tabular}{ll|lll|lll|llll}
\hline & & \multicolumn{4}{|c|}{$\operatorname{MSE}\left(\hat{\theta}_{0}\right)$} & \multicolumn{3}{c|}{$\operatorname{MSE}\left(\hat{\theta}_{1}\right)$} & \multicolumn{3}{c}{$\operatorname{MSE}\left(\hat{\theta}_{2}\right)$} \\
\hline$\alpha$ & $n$ & CL & NAIVE & ORACLE & CL & NAIVE & ORACLE & CL & NAIVE & ORACLE \\
\hline 1.1 & 200 & 0.0944 & 0.0767 & 0.0554 & 0.0209 & 0.0389 & 0.0331 & 0.0793 & 0.0567 & 0.0424 \\
& 400 & 0.0385 & 0.0507 & 0.0322 & 0.0124 & 0.0184 & 0.0180 & 0.0328 & 0.0387 & 0.0209 \\
& 600 & 0.0268 & 0.0416 & 0.0165 & 0.0096 & 0.0158 & 0.0143 & 0.0256 & 0.0429 & 0.0116 \\
\hline 2 & 200 & 0.1049 & 0.0955 & 0.0668 & 0.0195 & 0.0360 & 0.0379 & 0.0867 & 0.0556 & 0.0389 \\
& 400 & 0.0272 & 0.0538 & 0.0304 & 0.0104 & 0.0181 & 0.0163 & 0.0276 & 0.0431 & 0.0240 \\
& 600 & 0.0311 & 0.0485 & 0.0239 & 0.0080 & 0.0141 & 0.0134 & 0.0255 & 0.0376 & 0.0159 \\
\hline
\end{tabular}

Table 2: Bias, Variance and IMSE of functional coefficient estimators at $\tau=0.75$ in Case 1.

\begin{tabular}{ll|lll|lll|lll}
\hline & & \multicolumn{3}{|c|}{ CL } & \multicolumn{3}{c|}{ NAIVE } & \multicolumn{3}{c}{ ORACLE } \\
\hline$\alpha$ & $\mathrm{n}$ & Bias $^{2}$ & Var & IMSE & Bias $^{2}$ & Var & IMSE & Bias $^{2}$ & Var & IMSE \\
\hline 1.1 & 200 & 0.0439 & 0.2015 & 0.245 & 0.1240 & 0.0660 & 0.1900 & 0.0136 & 0.1176 & 0.1312 \\
& 400 & 0.0124 & 0.0812 & 0.0936 & 0.0862 & 0.0280 & 0.1142 & 0.0106 & 0.0486 & 0.0592 \\
& 600 & 0.0100 & 0.0594 & 0.0693 & 0.0770 & 0.0173 & 0.0943 & 0.0086 & 0.0296 & 0.0382 \\
2 & 200 & 0.1135 & 0.1841 & 0.2976 & 0.1802 & 0.0603 & 0.2405 & 0.0357 & 0.1818 & 0.2175 \\
& 400 & 0.0420 & 0.1172 & 0.1592 & 0.1114 & 0.0543 & 0.1656 & 0.0197 & 0.0913 & 0.1109 \\
& 600 & 0.0159 & 0.0831 & 0.0990 & 0.0840 & 0.0322 & 0.1162 & 0.0139 & 0.0428 & 0.0567 \\
\hline
\end{tabular}

Table 3: Empirical and theoretical standard errors for data generated in Case 1.

\begin{tabular}{llll}
\hline & $\mathrm{n}$ & Empirical SE & Theoretical SE \\
\hline$\theta_{0}$ & 200 & 0.279 & 0.248 \\
& 400 & 0.172 & 0.177 \\
& 600 & 0.166 & 0.147 \\
$\theta_{1}$ & 200 & 0.152 & 0.150 \\
& 400 & 0.109 & 0.106 \\
& 600 & 0.074 & 0.086 \\
$\theta_{2}$ & 200 & 0.258 & 0.220 \\
& 400 & 0.158 & 0.158 \\
& 600 & 0.147 & 0.131 \\
\hline
\end{tabular}


Table 4: Empirical coverage probabilities (in \%) of bootstrap confidence intervals with a nominal level of $95 \%$ for Case 1 at $\tau=0.5, \alpha=2$.

\begin{tabular}{lllll}
\hline$n$ & $\theta_{0}$ & $\theta_{1}$ & $\theta_{2}$ & $b(t)$ \\
\hline 200 & 97 & 99 & 94 & 95 \\
400 & 96 & 96 & 96 & 95 \\
600 & 95 & 98 & 96 & 93 \\
\hline
\end{tabular}

Table 5: Descriptive statistics for children obesity study. "Other" = Hispanics/Asians/Native Americans.

\begin{tabular}{lr}
\hline Variable & mean $(\mathrm{sd}) / \mathrm{N}(\%)$ \\
\hline log(BMI) & $2.846(0.159)$ \\
\hline Age & $8.378(0.754)$ \\
\hline Treatment & $134(58.261)$ \\
Control & $96(41.026)$ \\
\hline Blacks & $25(10.870)$ \\
Other & $42(18.260)$ \\
Whites & $163(70.870)$ \\
\hline Boys & $120(52.174)$ \\
Girls & $110(47.826)$ \\
\hline School 1 & $57(24.783)$ \\
School 2 & $89(38.696)$ \\
School 3 & $84(36.522)$ \\
\hline
\end{tabular}


Table 6: Parametric regression coefficient estimates by proposed (CL) and naive method at $\tau=0.2,0.5,0.8$. Standard errors from 500 boostrasp samples are included in parentheses, and significance at $5 \%$ level is in bold face.

\begin{tabular}{llllllllll}
\hline$\tau$ & Method & Inter & S1 & S2 & Trt & Sex & R1 & R2 & Age \\
\hline 0.2 & CL & $\mathbf{1 . 7 8 8}$ & -0.098 & -0.071 & -0.052 & -0.035 & 0.031 & 0.075 & 0.014 \\
& & $(0.267)$ & $(0.061)$ & $(0.040)$ & $(0.029)$ & $(0.034)$ & $(0.046)$ & $(0.074)$ & $(0.033)$ \\
& NAIVE & $\mathbf{2 . 4 9 3}$ & $\mathbf{- 0 . 0 5 4}$ & -0.019 & -0.013 & -0.025 & 0.010 & -0.009 & $\mathbf{0 . 0 3 5}$ \\
& & $(0.092)$ & $(0.022)$ & $(0.018)$ & $(0.015)$ & $(0.014)$ & $(0.018)$ & $(0.042)$ & $(0.011)$ \\
0.5 & CL & $\mathbf{2 . 5 3 9}$ & $\mathbf{- 0 . 0 6 9}$ & -0.023 & 0.005 & $\mathbf{- 0 . 0 4 4}$ & 0.039 & 0.049 & $\mathbf{0 . 0 4 0}$ \\
& & $(0.146)$ & $(0.024)$ & $(0.019)$ & $(0.019)$ & $(0.018)$ & $(0.023)$ & $(0.035)$ & $(0.017)$ \\
& \multirow{2}{*}{ NAIVE } & $\mathbf{2 . 5 6 2}$ & $\mathbf{- 0 . 0 6 9}$ & -0.038 & -0.000 & -0.032 & 0.020 & 0.048 & $\mathbf{0 . 0 3 6}$ \\
& & $(0.147)$ & $(0.028)$ & $(0.028)$ & $(0.026)$ & $(0.026)$ & $(0.029)$ & $(0.044)$ & $(0.017)$ \\
0.8 & CL & $\mathbf{3 . 0 5 8}$ & $\mathbf{- 0 . 1 4 3}$ & -0.043 & 0.000 & -0.037 & 0.017 & -0.029 & $\mathbf{0 . 0 9 3}$ \\
& & $(0.191)$ & $(0.040)$ & $(0.027)$ & $(0.027)$ & $(0.024)$ & $(0.033)$ & $(0.044)$ & $(0.024)$ \\
& \multirow{2}{*}{ NAIVE } & $\mathbf{2 . 7 8 5}$ & -0.080 & -0.049 & -0.024 & $\mathbf{- 0 . 0 7 1}$ & 0.004 & -0.011 & 0.029 \\
& $(0.163)$ & $(0.044)$ & $(0.031)$ & $(0.023)$ & $(0.027)$ & $(0.047)$ & $(0.072)$ & $(0.021)$ \\
\hline
\end{tabular}



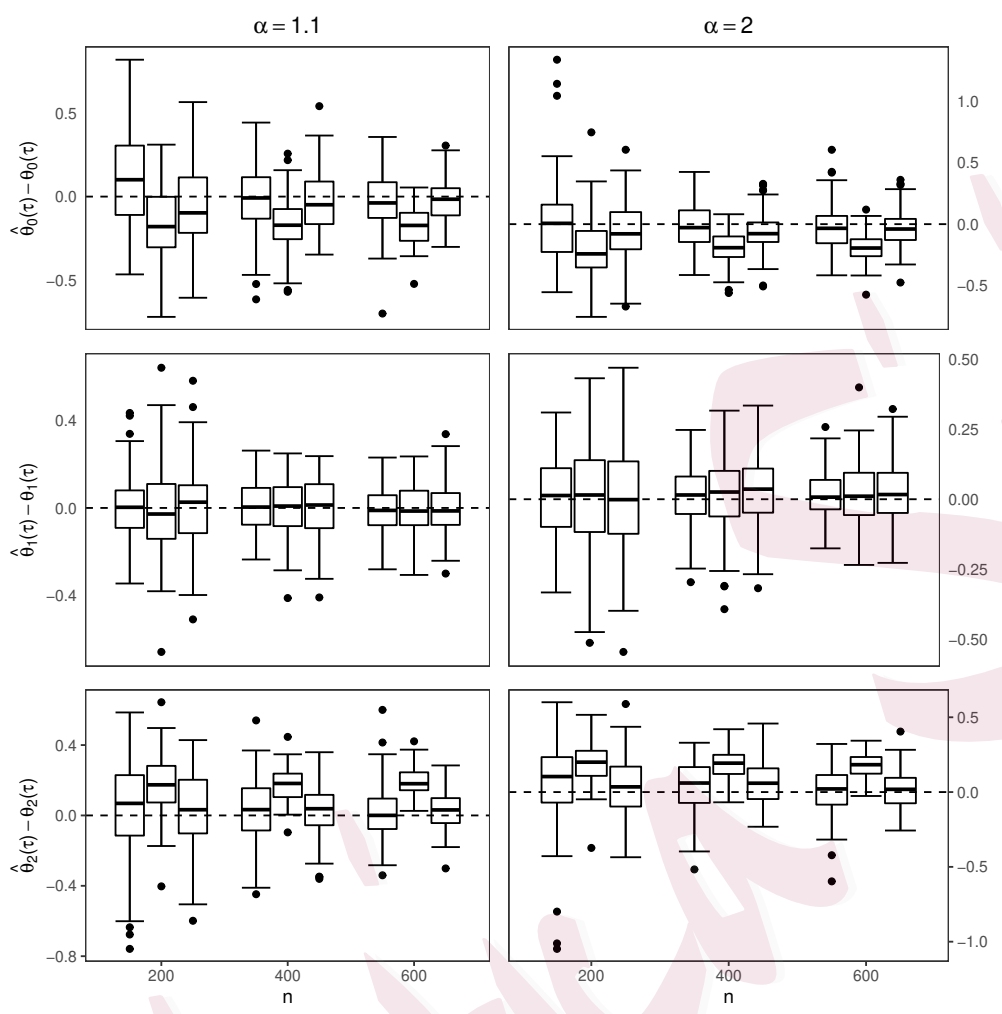

Figure 1: Boxplots of $\hat{\theta}_{k}(\tau)-\hat{\theta}_{k 0}(\tau), k=0,1,2$ at $\tau=0.75$, but for different $\alpha$ values and sample sizes with data generated in Case 1. In each figure, boxplots from left to right are in the order of CL, NAIVE and ORACLE. 

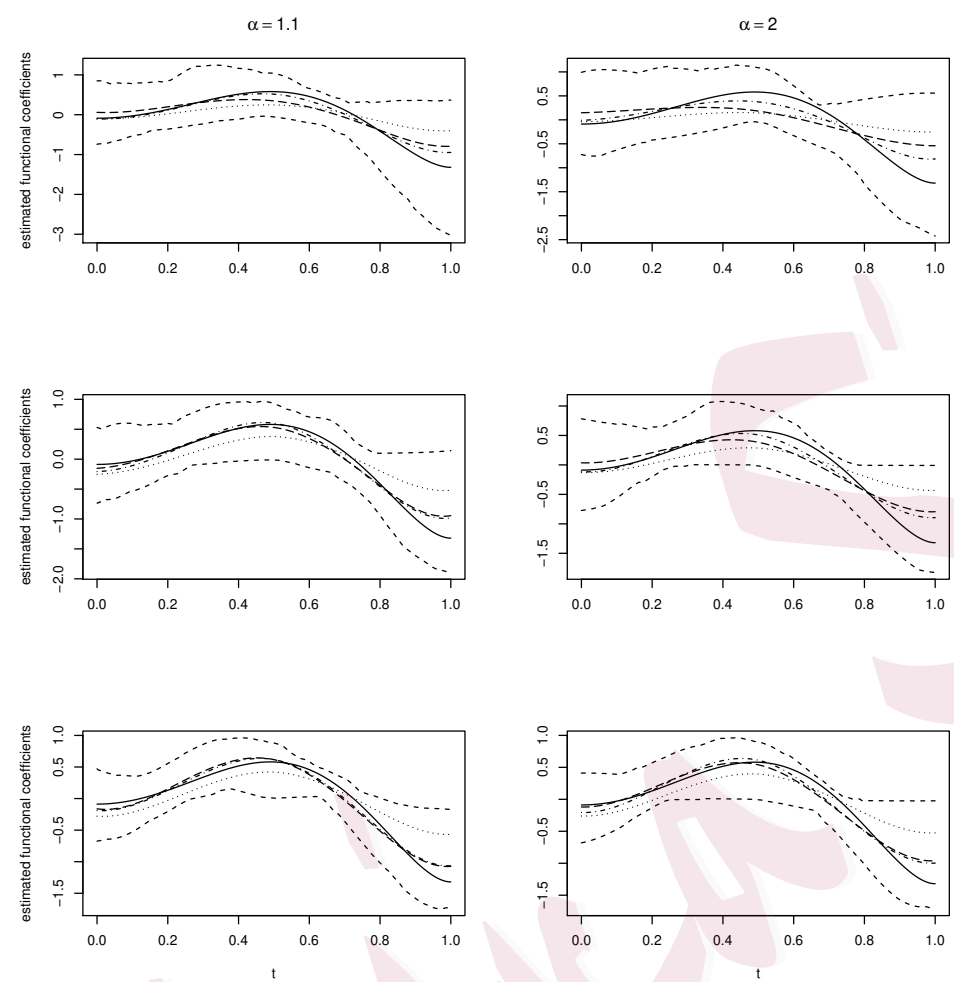

Figure 2: Estimated functional coefficients at $\tau=0.75$ for $\alpha=1.1$ or 2 with data generated in Case 1. Top to bottom are in the order of $n=200,400,600$ for each $\alpha$. In each plot, solid line represents true functional coefficient, dotted and dashed line for ORACLE, longer dashed line for CL, dotted line for NAIVE and shorter dashed lines for $95 \%$ interval of CL. 


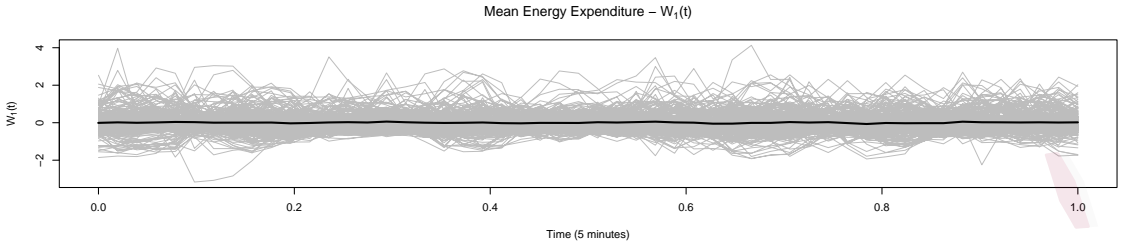

Mean Energy Expenditure - $W_{2}(t)$

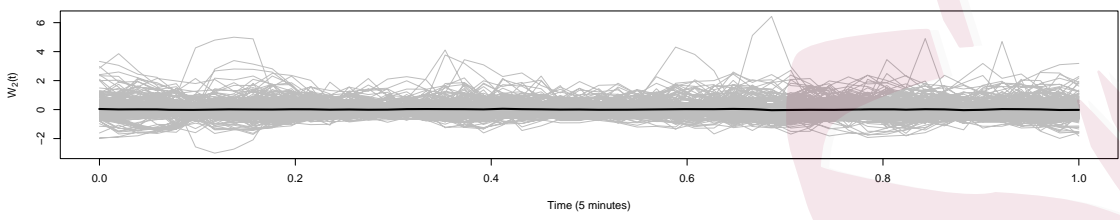

Mean Energy Expenditure $-W_{3}(t)$

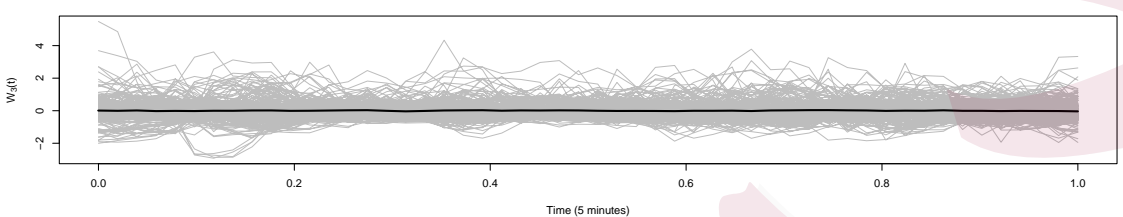

Figure 3: Plots of observed energy expenditure $\left\{W_{1}(t), W_{2}(t), W_{3}(t)\right\}$ versus time for all subjects at baseline.
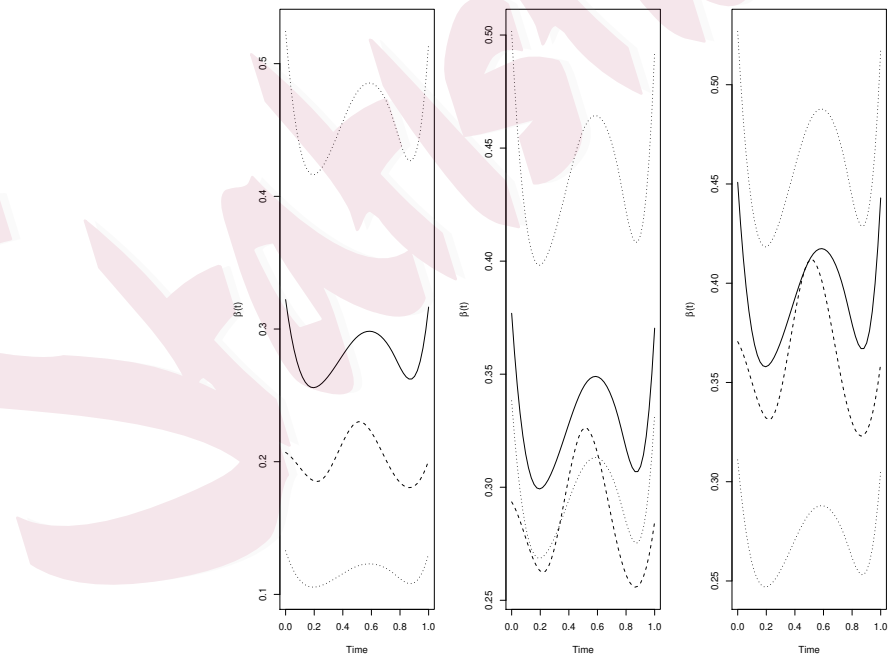

Figure 4: Plots of proposed (solid line) and naive (dashed line) estimates of $\beta(t)$ at $\tau=0.2,0.5,0.8$ from left to right. Two dotted lines are the $95 \%$ pointwise bootstrap confidence intervals with 500 boostrap samples. 


\section{REFERENCES}

\section{References}

Bassett, D. R. (2012) Device-based monitoring in physical activity and public health research. Physiological Measurement, 33, 1769. URL: http: //stacks.iop.org/0967-3334/33/i=11/a=1769.

Carroll, R. J., Ruppert, D., Stefanski, L. and Crainiceanu, C. (2006) Measurement Error in Nonlinear Models: A Modern Perspective, Second Edition. Chapman and Hall.

Crouter, S. E., Churilla, J. R. and Bassett, D. R. (2006) Estimating energy expenditure using accelerometers. European Journal of Applied Physiology, 98, 601-612.

Delaigle, A. and Hall, P. (2008) Using simex for smoothing-parameter choice in errors-in-variables problems. Journal of the American Statistical Association, 103, 280-287.

Firpo, S., Galvao, A. F. and Song, S. (2017) Measurement errors in quantile regression models. Journal of econometrics, 198, 146-164.

Fuller, W. (1987) Measurement Error Models. Chapman and Hall/CRC.

Geraci, M. and Bottai, M. (2014) Linear quantile mixed models. Statistics and Computing, 24, 461-479.

Hall, P. and Horowitz, J. L. (2007) Methodology and convergence rates for functional linear regression. Annals of Statistics, 35, 70-91.

He, X. and Liang, H. (2000) Quantile regression estimates for a class of linear and partially linear errors-in-variables models. Statistica Sinica, 129-140.

$\mathrm{Hu}, \mathrm{Y}$. and Schennach, S. (2008) Instrumental variable treatment of nonclassical measurement error models. Econometrica, 76, 195-216.

Jacobi, D., Perrin, A.-E., Grosman, N., Doré, M.-F., Normand, S., Oppert, J.-M. and Simon, C. (2007) Physical activity-related energy expenditure with the rt3 and tritrac accelerometers in overweight adults. Obesity, 15, 950-956.

Kato, K. (2012) Estimation in functional linear quantile regression. The Annals of Statistics, 40, 3108-3136.

Koenker, R. (2005) Quantile regression. Cambridge university press. 


\section{REFERENCES}

Koenker, R. and Bassett, Jr, G. (1978) Regression quantiles. Econometrica: journal of the Econometric Society, 33-50.

Kong, D., Xue, K., Yao, F. and Zhang, H. (2016) Partially functional linear regression in high dimensions. Biometrika, 103, 147-159.

Lu, Y., Du, J. and Sun, Z. (2014) Functional partially linear quantile regression model. Metrika, 77, 317-332.

Maffeis, C., Zaffanello, M., Pinelli, L. and Schutz, Y. (1996) Total energy expenditure and patterns of activity in 8-10-year-old obese and nonobese children. Journal of Pediatric Gastroenterology and Nutrition, 23, 256-261.

Nakamura, T. (1990) Corrected score function for errors-in-variables models: Methodology and application to generalized linear models. Biometrika, 77, $127-137$.

Rothney, M. P., Schaefer, E. V., Neumann, M. M., Choi, L. and Chen, K. Y. (2008) Validity of physical activity intensity predictions by actigraph, actical, and rt3 accelerometers. Obesity, 16, 1946-1952.

Shin, H. (2009) Partial functional linear regression. Journal of Statistical Planning and Inference, 139, 3405-3418.

Silverman, B. and Ramsay, J. (2005) Functional Data Analysis. Springer.

Stefanski, L. A. and Cook, J. R. (1995) Simulation-extrapolation: the measurement error jackknife. Journal of the American Statistical Association, 90, 1247-1256.

Tekwe, C., Zoh, R., Yang, M., Carroll, R., Honvoh, G., Allison, D., Benden, M. and Xue, L. (2019) Instrumental variable approach to estimating the scalar-onfunction regression model with measurement error with application to energy expenditure assessment in childhood obesity. Statistics in Medicine, 38, 37643781.

Wang, H. J., Stefanski, L. A. and Zhu, Z. (2012) Corrected-loss estimation for quantile regression with covariate measurement errors. Biometrika, 99, 405421. 


\section{REFERENCES}

Warolin, J., Carrico, A., Whitaker, L., Wang, L., Chen, K., Acra, S. and Buchowski, M. (2012) Effect of bmi on prediction of accelerometry-based energy expenditure in youth. Medicine and Science in Sports and Exercise, 44, 2428.

Wei, Y. and Carroll, R. J. (2009) Quantile regression with measurement error. Journal of the American Statistical Association, 104, 1129-1143.

Yao, F., Sue-Chee, S. and Wang, F. (2017) Regularized partially functional quantile regression. journal of multivariate analysis. Journal of Multivariate Analysis, 156, 39-56. 